\title{
Well-differentiated Papillary Mesothelioma With Invasive Foci
}

\author{
Andrew Churg, MD, ${ }^{*}$ Timothy Allen, MD, $\dagger$ Alain C. Borczuk, MD, $\ddagger$ Philip T. Cagle, MD, $\S$ \\ Francoise Galateau-Sallé, MD, /| Harry Hwang, MD, $\uparrow$ Bruno Murer, MD,\# Vundavalli V. Murty, MD, \\ Nelson Ordonez, MD, ** Henry D. Tazelaar, MD, †† and Mark Wick, MD $\$$
}

\begin{abstract}
Well-differentiated papillary mesotheliomas (WDPMs) are usually encountered as incidental findings in the peritoneal cavity in women. Most WDPMs are benign, and the histologic features that indicate a more aggressive course are controversial. We report 20 cases of WDPM, which contained invasive foci. Thirteen cases arose in the peritoneal cavity, 1 in a hernia sac, 3 in the pleural cavity, and 3 in hydroceles. The female:male ratio was $16: 4$, and age range was 7 to 74 years. Tumor was multifocal in 15 cases. Some tumors showed back-to-back papillae, a pattern mimicking invasion but discernible on pan-keratin stain as compressive crowding. True invasive patterns ranged from simple bland-appearing glands invading the stalks of the papillae to solid foci of invasive tumor of higher cytologic grade than the original WDPM. All 5 tested cases were negative for p16 deletion by fluorescence in situ hybridization, but $2 / 3$ had abnormal karyotypes. Recurrences were seen in 8 patients, and in 4 multiple recurrences were documented. Of 16 patients with follow-up, 14 are alive from periods of 6 months to 6 years (average $3.5 \mathrm{y}$ ), and 2 have known recurrent disease. One patient died of disseminated tumor at 8 years but without histologic confirmation of the nature of the tumor. We conclude that WDPM with invasive foci in the papillae appear to be prone to multifocality and recurrence, but that they rarely give rise to lifethreatening disease. We suggest that these lesions be called WDPM with invasive foci to alert clinicians to the possibility of recurrence.
\end{abstract}

From the *Department of Pathology, Vancouver General Hospital, Vancouver, BC, Canada; $\dagger$ Department of Pathology, The University of Texas Medical Branch Galveston; §Department of Pathology and Genomic Medicine, Houston Methodist Hospital; **Department of Pathology, The University of Texas MD Anderson Cancer Center, Houston, TX; \$Department of Pathology and Cell Biology, Columbia University Medical Center, New York, NY; $\uparrow$ PhenoPath Laboratories, Seattle, WA; † Department of Laboratory Medicine and Pathology, Mayo Clinic in Arizona, Scottsdale, AZ; ttDepartment of Pathology, University of Virginia Health System, Charlottesville, VA; \|Department of Pathology, CHU Caen, Caen, France; and \#Department of Clinical Pathology, Ospedale dell'Angelo Venezia, Mestre, Italy.

Conflicts of Interest and Source of Funding: The authors have disclosed that they have no significant relationships with, or financial interest in, any commercial companies pertaining to this article.

Correspondence: Andrew Churg, MD, Department of Pathology, Vancouver General Hospital, 910 W 10th Ave, Vancouver, BC, Canada V5Z 1M9 (e-mail: achurg@interchange.ubc.ca).

Copyright (C) 2014 by Lippincott Williams \& Wilkins
Key Words: well-differentiated papillary mesothelioma, malignant mesothelioma, p16 deletion

(Am J Surg Pathol 2014;38:990-998)

W ell-differentiated papillary mesotheliomas (WDPMs) are characterized by a mesothelial proliferation with a papillary architecture, a myxoid appearance to the papillary cores, and a single layer of cytologically bland mesothelial cells covering the papillae. ${ }^{1}$ Most WDPMs are found in the peritoneum in women, but they also occur in the pleural cavity, pericardium, and tunica vaginals.

The neoplastic versus non-neoplastic nature of WDPM and the question of how these lesions behave has always been somewhat controversial. WDPMs are sometimes multifocal and occasionally recur after surgical excision, but, at least in the peritoneal cavity, neither multifocality nor recurrence has clearly been associated with an adverse prognosis. ${ }^{2,3}$ A related question is whether WDPMs ever give rise to diffuse malignant mesothelioma or whether these are completely separate lesions. This issue has been raised in various papers, but very few verifiable cases of WDPM developing into malignant mesothelioma have been reported (see the Discussion section).

In this paper, we report a series of 20 WDPMs containing invasive foci, look at the effect of such foci on recurrence and survival, and ask whether there is any clear relationship between WDPM and malignant mesothelioma.

\section{METHODS}

Cases of possible WDPM with invasion were selected from the authors' consult files. Thirty-one cases were submitted for review by all the authors, and after initial review 20 were accepted as true WDPM with invasive foci. All tumors had a largely papillary architecture, with the papillae being formed of myxoidappearing cores and a covering composed of a single layer of relatively bland-appearing mesothelial cells. Invasive foci or higher-grade tumor within the overall papillary structures was considered a part of the entity of WDPM with invasive foci, but areas of solid tumor outside the 
papillary structures were viewed as diffuse malignant mesotheliomas and were not accepted as WDPM. In 1 case (case 13) there was a small invasive focus in the fat under the WDPM. Immunohistochemical staining results showing a mesothelial origin were available for all except 2 of the cases. Clinical features including location, multifocality, recurrence, survival, and follow-up looking for evidence of diffuse malignant mesothelioma were recorded. This study was approved by the University of British Columbia Committee on Human Research Ethics.

\section{p16 Fluorescence In Situ Hybridization Testing}

In 5 cases, p16 fluorescence in situ hybridization (FISH) testing was performed to look for p16 deletion. Briefly, 4-mm-thick formalin-fixed paraffin-embedded sections were deparaffinized and pretreated using a VP2000 processor (Abbott Laboratories). After deparaffinization and rehydration, the slides were acid treated in $0.2 \mathrm{~N} \mathrm{HCl}$ for 20 minutes, washed in $2 \mathrm{X} \mathrm{SSC}$, placed in $8.1 \%$ sodium thiocyanate, washed in $2 \mathrm{X} \mathrm{SSC}$, digested in $0.8 \%$ pepsin for 10 minutes, washed in $2 \mathrm{X}$ SSC, and postfixed in $10 \%$ neutral buffered formalin. Slides were then denatured for 16 minutes at $79^{\circ} \mathrm{C}$ and then hybridized with a CDKN2A/CC9 probe (Cymogen Diagnostics) that was diluted $1: 10$ in Denhyb probe hybridization buffer (Insitus) and then incubated overnight (14 to $16 \mathrm{~h}$ ) at $37^{\circ} \mathrm{C}$. The Cymogen CDKN2A/CC9 probe comprises a $298 \mathrm{~kb}$ orange fluor-labeled probe to 9p21.3 and a green fluor-labeled probe to the centromeric region of chromosome 9 (9p11.1-q11.1). After incubation, slides were washed for 3 minutes in $0.4 \mathrm{X} \mathrm{SSC} / 0.3 \% \mathrm{NP} 40$ at $79^{\circ} \mathrm{C}$, followed by washing at $25^{\circ} \mathrm{C}$ in $2 \mathrm{X}$ SSC for 3 minutes, and quick rinse in $\mathrm{dH}_{2} \mathrm{O}$. After air drying, slides were counterstained using DAPI/Fluorguard solution and then coverslipped. Slides were then scanned using a Metasystems slide scanner (MetaSystems, Altlussheim, Germany). A positive homozygous deletion event is defined as a DAPI-stained nucleus with no orange signals and at least 1 green signal. Using the Metasystems captured images, at least 50 to 100 nuclei were counted for each case, and the percentage of positive nuclei was calculated. The threshold, to account for section truncation artifact, for p16 homozygous deletion for this assay on formalin-fixed paraffin-embedded sections is $12 \%$.

\section{Karyotype Analysis}

Chromosome analysis was performed using standard methods. Briefly, fresh tumor specimens collected after surgery were washed with an antimycotic $(0.5 \mu \mathrm{g} / \mathrm{mL}$ Amphotericin B) and antibiotics $(200 \mathrm{IU} / \mathrm{mL}$ penicillin and $200 \mu \mathrm{g} / \mathrm{mL}$ streptomycin), minced, and digested overnight with collagenase $(200 \mathrm{U} / \mathrm{mL})$ in complete DMEM medium. Dissociated cells were washed twice and then cultured in complete DMEM medium supplemented with epidermal growth factor and insulin-transferrin-sodium selenate (Life Technologies). The cultures were monitored daily, and appropriately confluent cells were subjected to metaphase preparations using standard methods after addition of colcemid for 12 hours. Metaphase preparations were subjected to GTG banding and analyzed by standard methods. The karyotypes were described by standard cytogenetic nomenclature.

\section{RESULTS}

\section{Demographic and Clinical Features}

Twenty cases were accepted on review as showing an underlying pattern of WDPM with invasive foci. Table 1 shows demographic data and lists the sites and sizes of the lesions. There were 16 women and 4 men with an age range from 7 to 74 . The majority of cases (13) arose in the peritoneal cavity, whereas 1 developed in a hernia sac, 3 in the pleural cavity, and 3 in hydrocele sacs.

\section{Pathologic Findings}

In 15 cases the lesions were multifocal. Tumor sizes ranged from $<1$ to $12 \mathrm{~cm}$. Although all tumors showed a typical microscopic pattern of WDPM (Figs. 1A, 2A, $\mathrm{C}, 3 \mathrm{~A}, 4 \mathrm{~A})$, in 6 cases the lesions had undergone a phenomenon that we view as compressive crowding; that is, the usual spaced papillae characteristic of WDPM (Fig. 1A) were replaced by back-to-back papillae (Figs. 1A, B), an appearance that at first glance mimicked an invasive tumor. However, keratin stains (Fig. 1C) showed clearly that outlines of the original papillae were still visible. Lesser degrees of compressive crowding than shown in Figures $1 \mathrm{~A}-\mathrm{C}$ were seen in some of the other cases. In all cases the papillae were covered by a single layer of flattened to cuboidal cells, and in some cases these cells had prominent nucleoli; however, multilayering of the covering cells and marked cytologic atypia were never present.

Immunohistochemical staining information was available for $18 / 20$ cases and showed a typical pattern of mesothelial markers (Table 2). One case (case 19) was an incidental microscopic finding and was cut through on initial hematoxylin and eosin, therefore immunostaining could not be performed, and for 1 case (case 6) immunostaining results were not available.

Invasive foci always constituted a small area of the lesion. A wide variety of invasive patterns was seen (Figs. 1D-F, 5A, B, 2B, D, 3B). These included small bland-appearing mesothelial glands in a fibrotic stroma, solid or near solid areas of bland mesothelial cells (Figs. 1F, 5A, B, 2D), solid sheets of spindled cells (Fig. 2B), and, much less frequently, cytologically highergrade lesions appearing as sheets of atypical mesothelial cells or atypical cells forming glands (Figs. 1D, E, 3B). For the most part the invasive foci tended to be superficial and were confined to the polyp that constituted the WDPM lesion, but in 1 case (case 13) they invaded fat (Fig. 4). In 1 case (case 10) several separate invasive foci were present.

p16 FISH testing was performed on the invasive foci in 5 cases (Table 2), and none showed p16 deletion. Karyotyping was successfully performed on 3 cases (Table 2); because the invasive foci were only microscopic findings, the 
TABLE 1. Demographic and Clinical Data

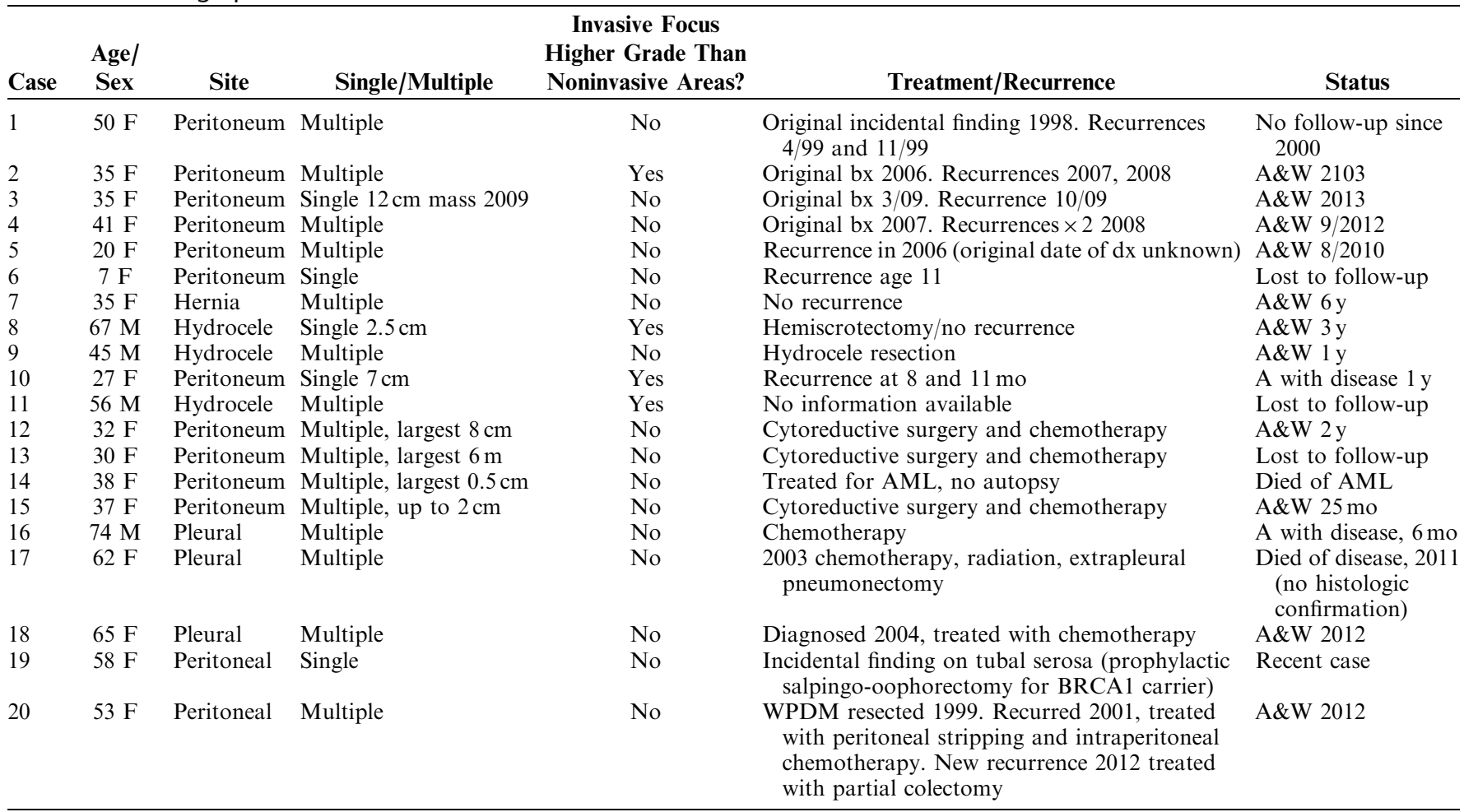

A indicates alive; AML, acute myelogenous leukemia; A\&W, alive and well.

karyotypes were derived from the whole WDPM lesions. Of these, 2 cases revealed clonal abnormalities, whereas the third case yielded a normal karyotype. Of the 2 cases with nonrandom chromosome aberration, 1 showed 46,XX, del(14)(q12q24),t(15;16)(q22;p13.3), add(21)(q22) in 7 of 16 metaphases analyzed. The other tumor showed 46,XX,-4, + del(6)(q13), del(10(p11.2),-22, + $\operatorname{mar}[\mathrm{cp} 8]$.

\section{Follow-up}

A number of patients were treated by cytoreductive surgery and chemotherapy, whereas in the remainder recurrent lesions were simply removed. Follow-up data were available for 16 patients. Recurrence of WDPM was seen in 8 patients, and in 4 of these patients there were multiple recurrences (Table 1). Fourteen patients are alive at the last follow-up (range 6 mo to $6 \mathrm{y}$, mean $3.5 \mathrm{y}$ ). Two patients are alive with apparently recurrent but nonprogressive disease. One patient died of acute myelogenous leukemia. In 1 patient (case 17) there was clinical evidence of disseminated disease leading to death after 8 years. However, no histologic confirmation of the nature of the disseminated disease was available (see the Discussion section).

\section{DISCUSSION}

WDPM continues to be an enigmatic lesion, with opinions about its nature running the gamut from a nonneoplastic reactive process to a benign tumor, a low-grade neoplasm or neoplasm of uncertain malignant potential, or a form of malignant mesothelioma; indeed sometimes these cases are treated clinically as if they were malignant mesotheliomas, as is evident in Table 1.

Both clinical behavior and (limited) molecular analyses suggest that WDPMs are in fact neoplastic. Ribeiro et $\mathrm{al}^{7}$ reported 2 cases in sisters that they believed represented WDPM and that showed BAPI mutations, something that has been described in unequivocal diffuse malignant mesotheliomas. ${ }^{6,8}$ On review their case 1 appears to us to actually be an ordinary malignant mesothelioma, but case 2 is most likely a WDPM with invasion, although the possibility that this is really an ordinary diffuse malignant mesothelioma with a papillary area cannot be ruled out.

In the present study, none of the 5 cases analyzed had p16 deletions, another common finding in diffuse malignant mesotheliomas, ${ }^{9}$ but 2 of 3 tumors attempted for karyotype exhibited clonal chromosome aberrations. Chromosome abnormalities in malignant tumors arising from the pleura are generally complex and involve almost all chromosomes (http://cgap.nci.nih.gov/Chromosomes/ RecurrentAberrations). No specific chromosome translocations have been reported in mesotheliomas. The most frequent aberrations are monosomies, deletions, or gains of chromosomes. The common chromosome losses are $22,13,14$, and 4 , gain of chromosome 7 , and deletion involving $1 \mathrm{p}, 3 \mathrm{p}, 6 \mathrm{q}$, and $9 \mathrm{p}$. One of our cases showed 

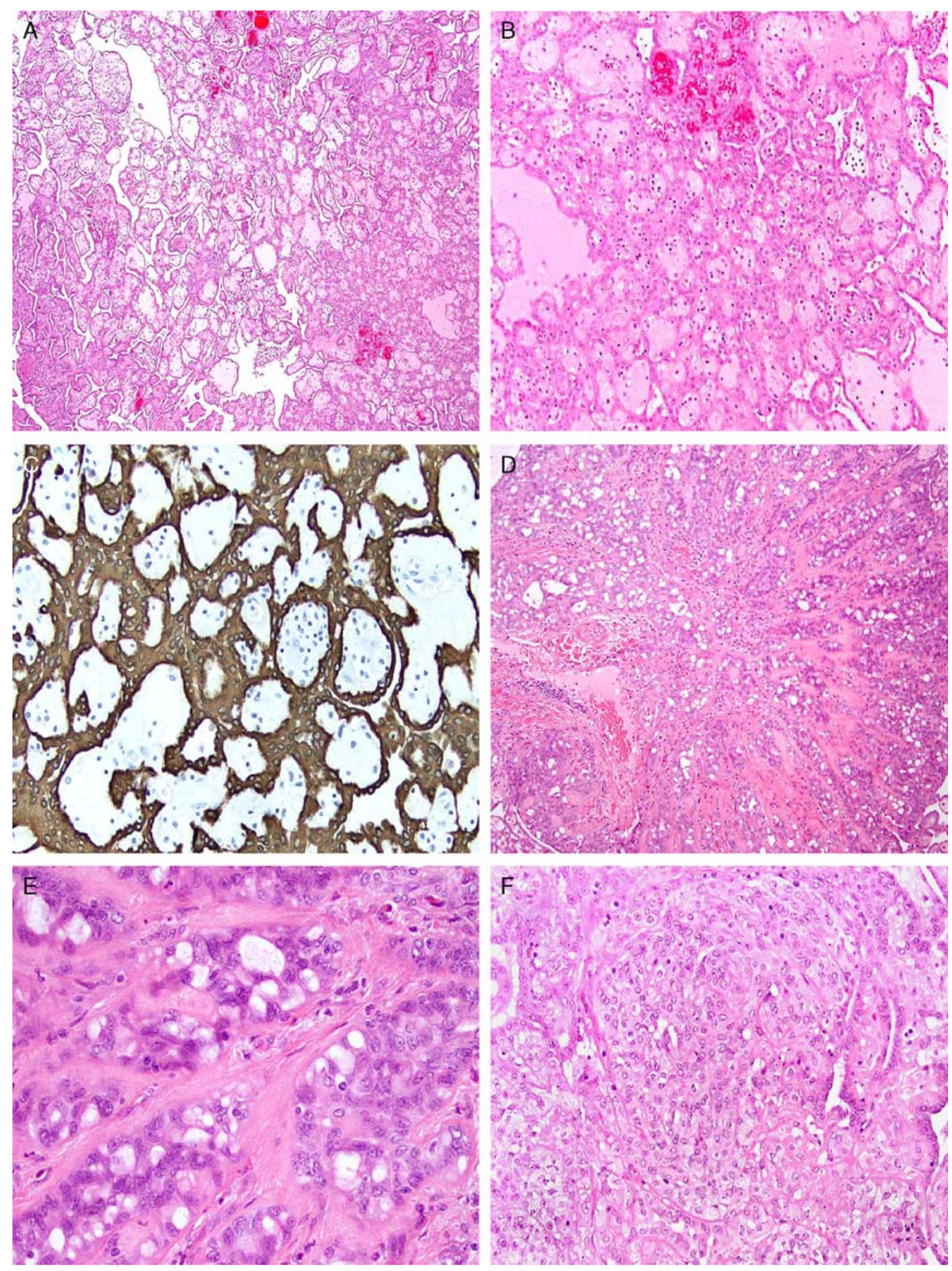

FIGURE 1. Peritoneal WDPM with areas of compressive crowding and invasive foci (case 10). A, Typical area of WDPM with myxoid-appearing papillae covered by a single layer of mesothelial cells in the center of the field and compressive crowding at the right hand edge. B, Another area of compressive crowding at higher power; this appearance leads to a false impression of invasive tumor. C, Pan-keratin stain outlines the retained papillary structure in the areas of crowding. D and E, Invasive focus with a somewhat complicated architecture and higher cytologic grade. F, Another invasive focus with a solid nodule of bland epithelial mesothelial cells. Taken by themselves these invasive foci are indistinguishable from diffuse malignant mesothelioma. 

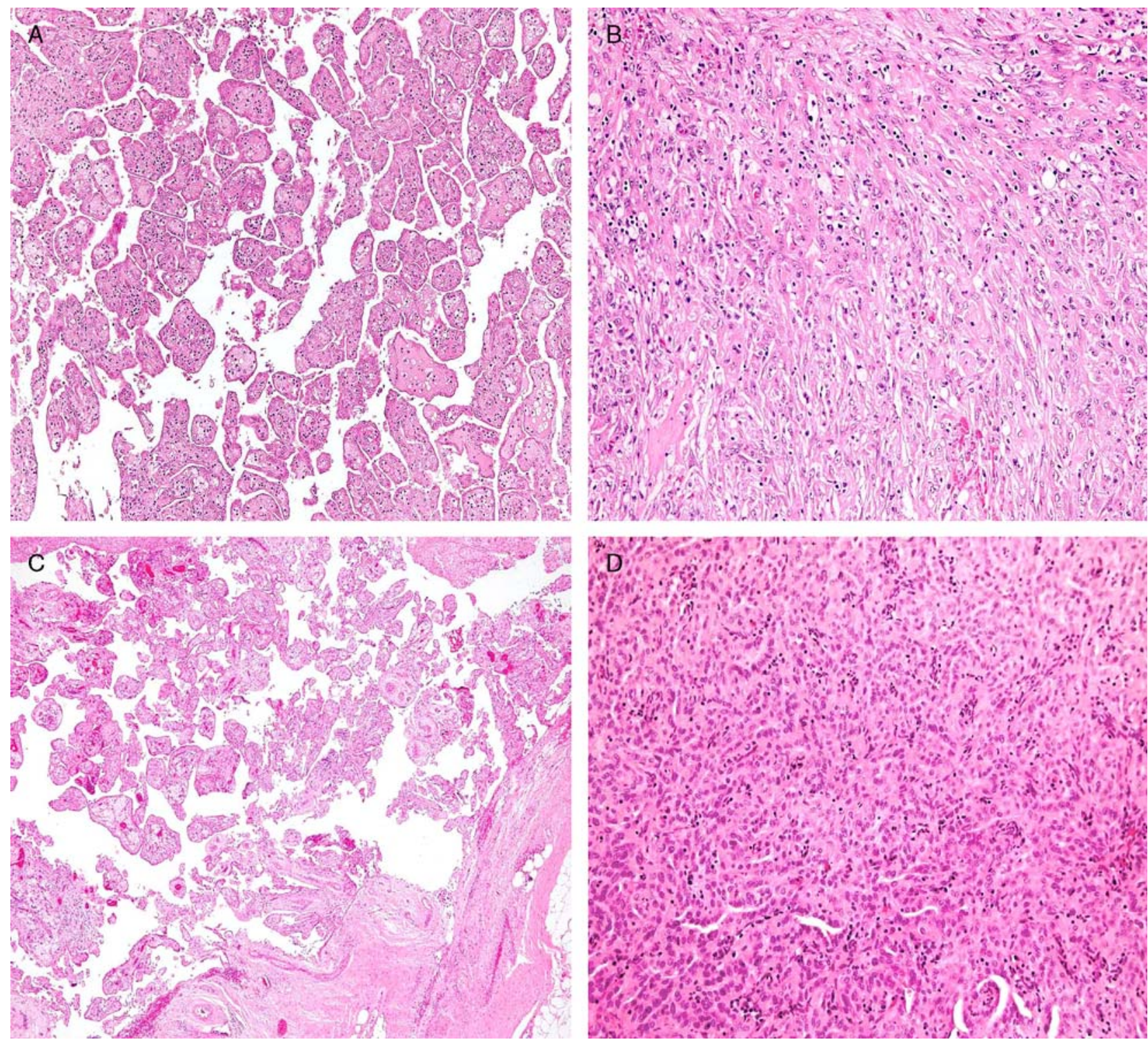

FIGURE 2. A WDPM arising in the peritoneum (case 2). Original tumor in the peritoneum (A and B) and recurrent tumor 1 year later ( $C$ and $D)$. Low-power views show typical WDPM patterns ( $A$ and $C$ ), but in (B) there is an invasive focus of proliferating spindle cells, and in (D) an invasive focus composed of compact simple tubules.

losses of chromosomes 4, 22, and 6q deletion. The second case showed deletion of a large part of $14 \mathrm{q}$, which is equivalent to loss of 14 . Thus, both the chromosomally abnormal karyotypes that we obtained from WDPM showed similarities to chromosome changes seen in pleural mesotheliomas. Translocations in $15 \mathrm{q} 22$ and 16 p13.3 have been described in hematologic malignancies and rare solid tumors, but the specific translocation between 15 and 16 seen here has not been previously described. ${ }^{10}$

The fact that WDPM can recur also favors a neoplastic process. Information on recurrence is difficult to glean from the literature, but in the recent Malpica et $\mathrm{al}^{2}$ study recurrence was observed in only 1 of 22 patients.
Interestingly, Malpica and colleagues described areas that we would call low-grade invasive foci in 15 of 26 patients. In contrast, the present series has a higher rate of recurrence ( 8 of 20 cases), and, in several cases, multiple recurrences, which leads us to suggest that WDPM with invasive foci may be particularly prone to recurrence.

Part of the problem in understanding the behavior of WDPM lies in the morphologic definition. The series of cases reported by Daya and $\mathrm{McCaughey}^{3}$ is usually viewed as the first clear description of these lesions, but in fact the Daya and McCaughey cases, although described as being papillary in part in all cases, also had a broad range of other patterns. The one papillary area illustrated by Daya and McCaughey is not all that similar to the 

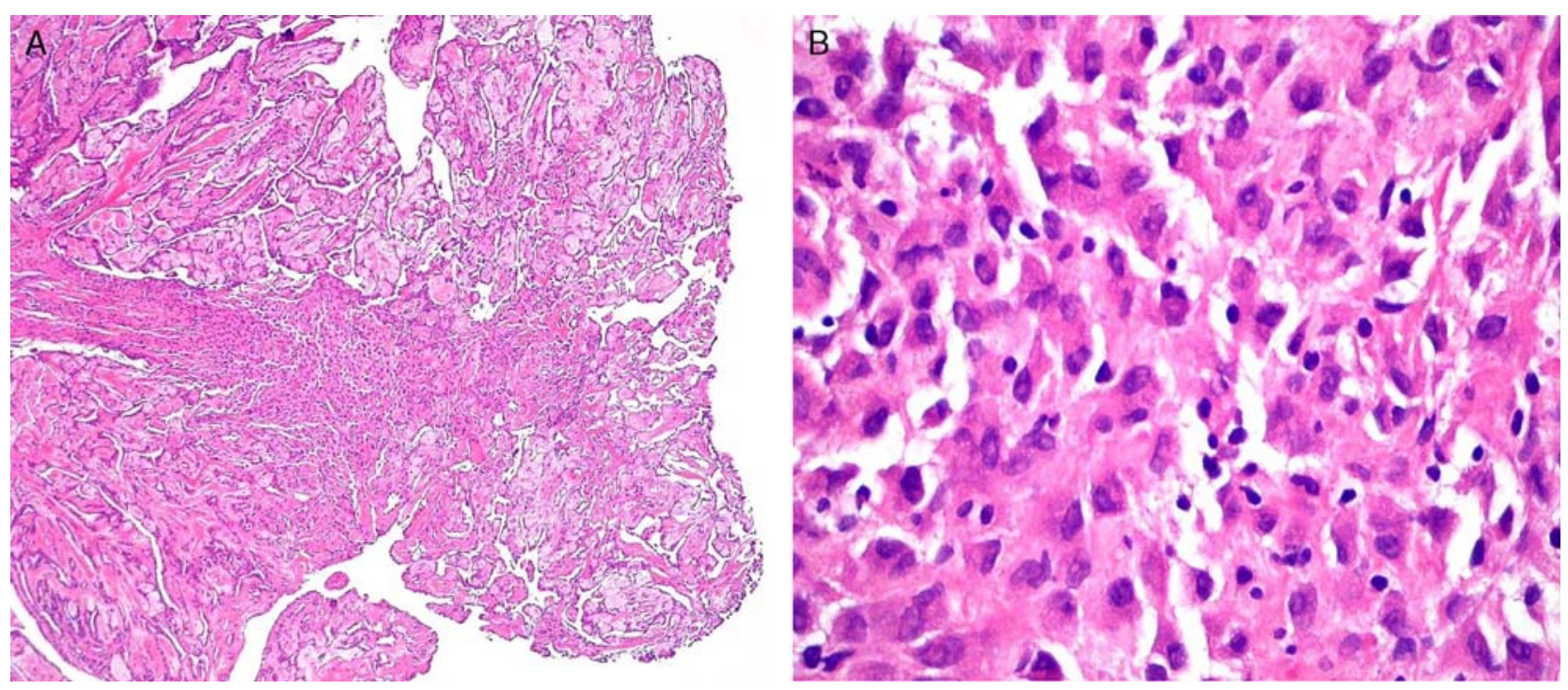

FIGURE 3. Invasive focus arising in a WDPM in a hydrocele (case 8). Low power (A) shows a typical WDPM architecture but with a solid invasive focus in the center; this focus is shown at higher power in (B). Note the higher cytologic grade.

lesion currently accepted by most authors as WDPM, and diagnostic criteria for WDPM appear to have slowly changed over time.

More recent descriptions of WDPM have all emphasized the rather specific papillary component with more or less myxoid and typically fairly plump cores with a single layer of overlying bland mesothelial cells as the essential feature of WDPM, ${ }^{4,11-19}$ even though in some instances different names have been applied to the lesion. Goldblum and Hart ${ }^{14}$ simply referred to them as "localized mesotheliomas," and Brimo et al ${ }^{12}$ called them "mesotheliomas of uncertain malignant potential."
Whether WDPM can transform into lesions of higher malignant potential, including diffuse malignant mesotheliomas, is a controversial issue. One of the confounding factors in this regard is that large WDPM can undergo what we regard as compressive crowding of the papillae (Fig. 1). Some reports have viewed such areas as cribriform (potentially invasive) patterns and possibly indicative of a worse prognosis (eg, Brimo et al ${ }^{12}$ and Trpkov et $\mathrm{al}^{16}$ ), and the term "mesothelioma of uncertain malignant potential" has been applied to such lesions, ${ }^{12}$ but what this really appears to be is compression of the papillae together, leaving lines of what

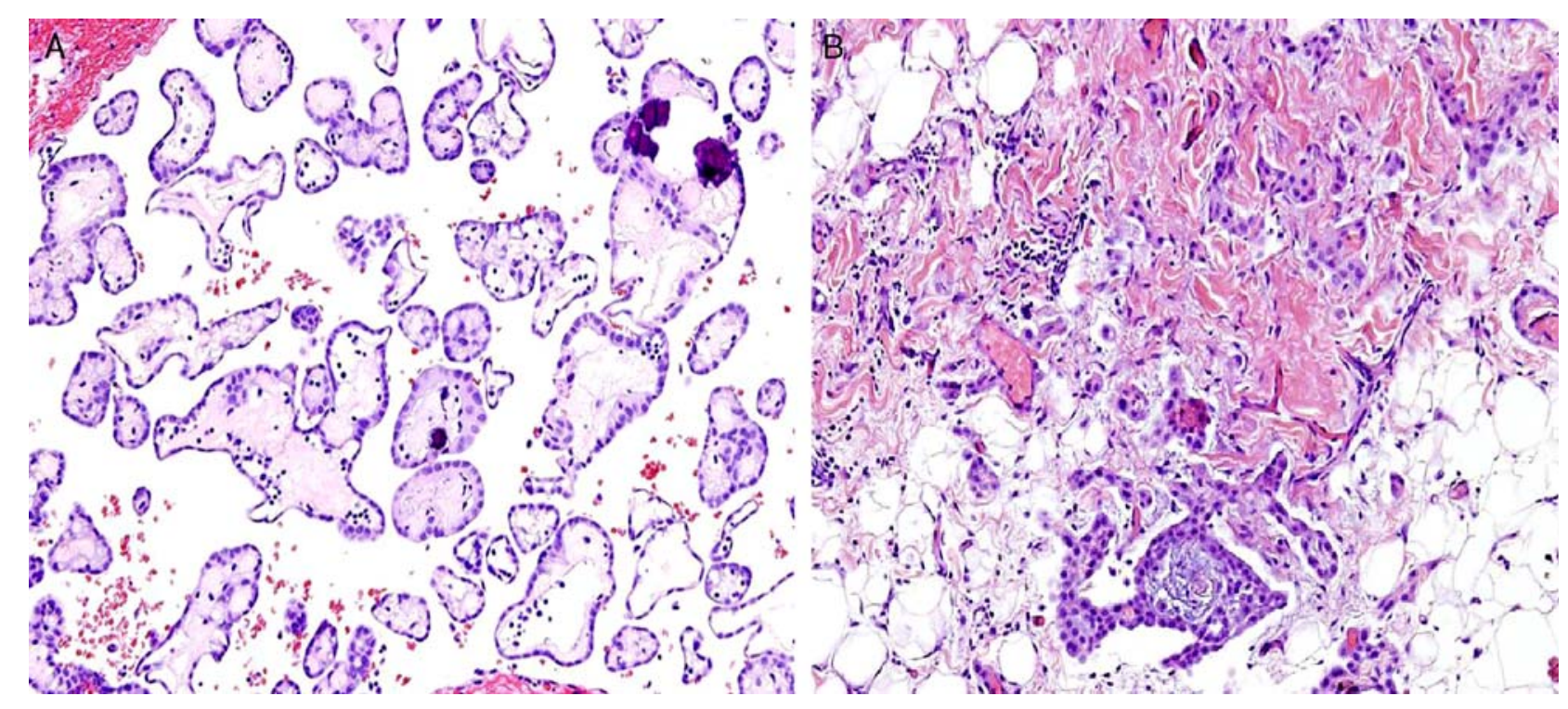

FIGURE 4. Invasion of the fat in a peritoneal WDPM (case 13). A, Typical picture of WDPM. B, An area in which bland-appearing mesothelial cells invade the fat underneath the tumor. 
TABLE 2. Immunohistochemical and Molecular Data

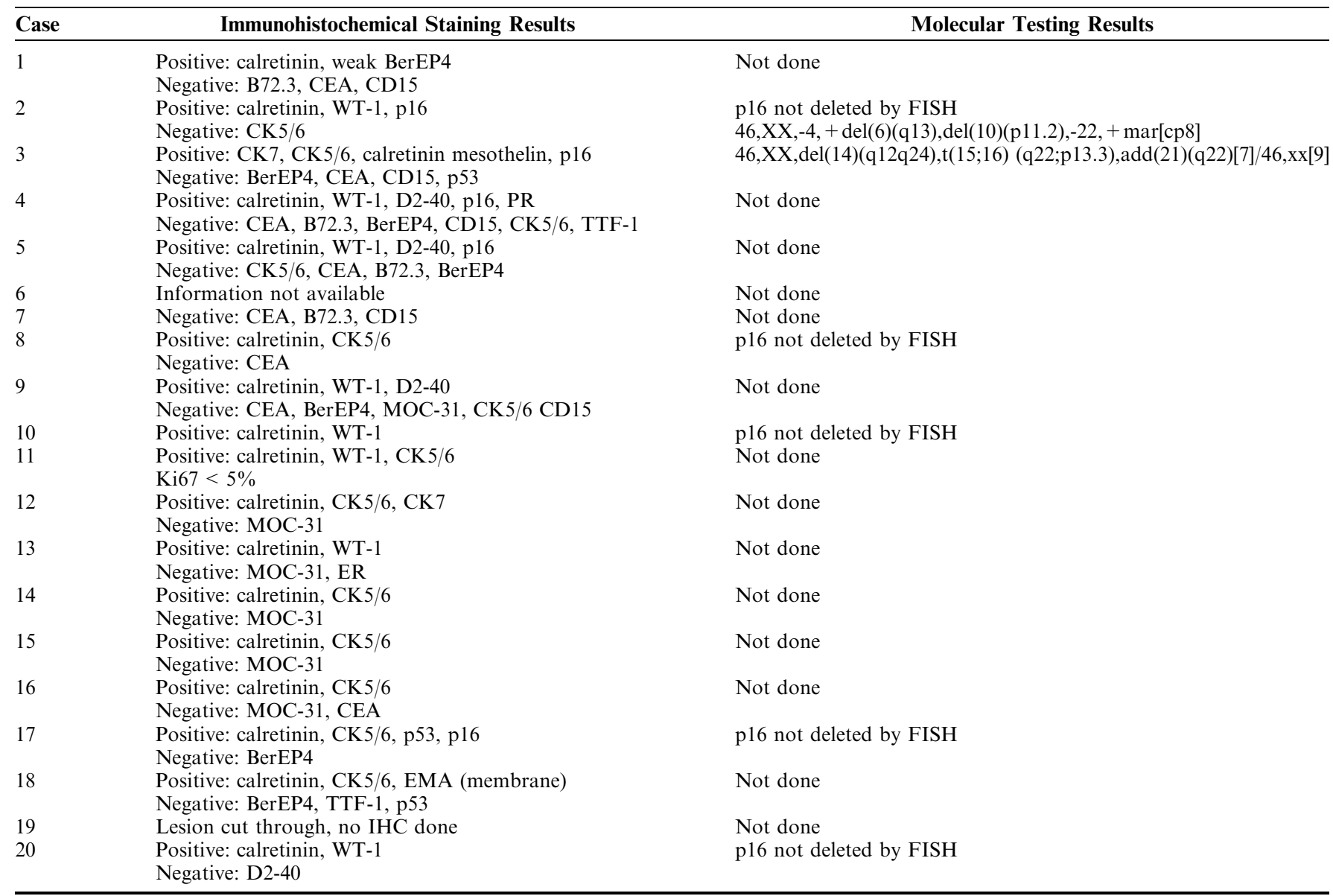

were originally surface mesothelial cells producing a false impression of "invading" stroma. Keratin stains show that the original papillary structure with covering mesothelial cells is still intact and that this pattern is not invasion (Fig. 1). It is possible that labeling such cases as true malignant mesotheliomas may account in part for the far better survivals seen in peritoneal compared with pleural mesotheliomas, even, sometimes, with what would appear to be inadequate therapy for a malignant mesothelioma. ${ }^{5}$

Nonetheless, there are morphologic changes in WDPM that we believe have to be regarded as true invasion, even though the original authors did not regard them as such. For example, the original Daya and McCaughey $^{3}$ paper illustrates a pattern that is fundamentally identical to an epithelial mesothelioma with a tubulopapillary pattern, and a similar picture is shown by Malpica et $\mathrm{al}^{2}$ (their Fig. 1F).

Daya and $\mathrm{McCaughey}^{3}$ contended that these were extremely bland cells and hence not malignant, but cytologic grade is a poor indicator of malignancy in epithelial mesotheliomas, many of which are remarkably bland appearing. Malpica et $\mathrm{al}^{2}$ also showed an image of glands from a WDPM invading the ovarian cortex (their Fig. 3); it is hard to see how such patterns cannot be viewed as malignant. The same is true of areas such as our Figures. 1D-F, 5A, B, 2B-D, 3B, and 4B. We suggest, however, that when such areas are low grade and localized to the papillae of WDPM, even though they morphologically mimic malignant mesotheliomas (albeit in very small areas), they generally do not give rise to diffuse malignant mesotheliomas. However, they may predispose to multifocality and recurrence. Indeed, it is striking that, in the cases in which we had material available for review from recurrences, the recurrences of these tumors had the morphology of WDPM and not malignant mesotheliomas (eg, Figs. 2A, C).

Most reports in the literature on malignant mesothelioma developing in WDPM either lack convincing illustrations (eg, Washimi et al, ${ }^{20}$ who are illustrating reactive mesothelial hyperplasia and not WDPM) or have such a long time interval between the diagnosis of WDPM and mesothelioma (eg, $10 \mathrm{y}$ in the 2 cases reported by Galateau-Salle et $\mathrm{al}^{13}$ ) that it is difficult to be sure that the WDPM and subsequent malignant mesothelioma are connected. One of our cases had an 

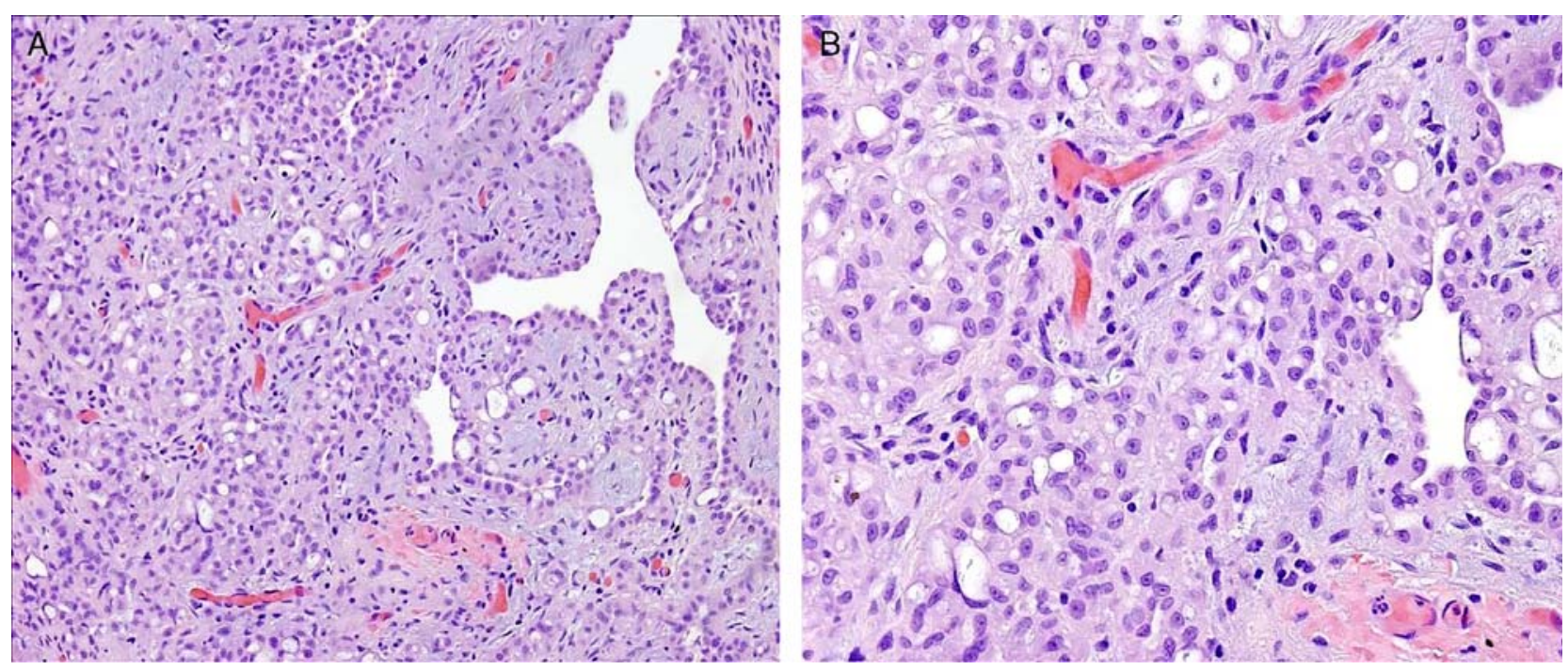

FIGURE 5. Invasive foci in a WDPM arising in the peritoneum at medium (A) and high (B) power (case 14). In a given field, these patterns of growth mimic diffuse malignant mesotheliomas.

extrapleural pneumonectomy but died of disseminated disease after 8 years (case 17). Unfortunately we have not been able to retrieve the pathology samples from the extrapleural pneumonectomy, so the nature of the progressive tumor is not known.

There are a few examples in the literature in which there does appear to be a connection between WDPM and a subsequent malignant mesothelioma. Torii et a ${ }^{15}$ reported a multifocal WDPM in the pleura with clear invasion of the lung and chest wall in the extrapleural pneumonectomy specimen, but, interestingly, no recurrence was reported up to 8 months. Bürrig 18 described a man with multiple peritoneal WDPM on biopsy who developed ascites 1 year later and died of a diffuse malignant mesothelioma of the peritoneum 5 years later, and Butnor et $\mathrm{a}^{11}$ reported a man with a peritoneal WDPM who developed radiologically progressive disease and died 3 years later but unfortunately without autopsy confirmation of the diagnosis.

We believe that several of the cases in this series do show a potential connection between WDPM and malignant mesothelioma, but for the most part these were represented by focal areas of higher cytologic grade and/ or architectural complexity (Figs. 1D, E, 3B). In case 13, there was invasion of underlying fat (Fig. 4B), which was unequivocal evidence of malignancy. Unfortunately this case was lost to follow-up. The case illustrated in Figure 1 had rapid recurrence, but the recurrent lesions were widely separated, rather than having the appearance of a diffuse malignant mesothelioma. The case shown in Figure 3 had no evidence of recurrence at 3 years at hemiscrotectomy. These findings suggest that even in the cases in which the morphology mimics that of a malignant mesothelioma, the functional behavior appears to be low grade. It appears preferable to label such cases as WDPM with invasive foci rather than malignant mesotheliomas.
It is important to emphasize the differential diagnosis of WDPM with or without invasion. A particular problem is ordinary diffuse malignant mesotheliomas that are partially papillary; if a biopsy samples only the papillary area, such a tumor can mimic WDPM. Often review of the operative report will solve the problem, as diffuse malignant mesotheliomas typically show either multiple obviously malignant nodules or a confluent rind of tumor on the serosal membranes, rather than the more delicate and often translucent foci of WDPM. In addition, tumors with WDPM-like papillary foci but obvious mesothelioma outside the papillae should be regarded as malignant mesotheliomas. Our data on the lack of p16 deletion in WDPM suggest that evaluation of p16 by FISH may also be of use in defining cases that are malignant mesotheliomas, but we make that comment with caution because of the small number of cases that were analyzed by FISH and also because only a proportion of diffuse malignant mesotheliomas show p16 deletion. ${ }^{9}$

Reactive mesothelium can also appear papillary, but generally such papillae are small and lack any kind of core or have fibrovascular cores rather than the myxoid cores of WDPM. WDPMs, by contrast, always have a distinctly papillary architecture with myxoid cores and a single layer of cytologically bland mesothelial cells covering the papillae and maintain this fundamental structure, even if the tumor is several centimeters in diameter.

In summary, this study suggests that WDPMs with invasive foci within the papillae have a distinct tendency toward multifocality and also toward recurrence and that such recurrences may be frequent in a given patient. Arguably the invasive foci represent early malignant mesothelioma in some cases, but because the development of overt diffuse malignant mesothelioma in this setting seems to be uncommon, we suggest calling these lesions WDPM with invasive foci. 


\section{REFERENCES}

1. Travis WD, Brambilla E, Muller-Hermelink HK, et al. Tumors of the Lung, Pleura, Thymus, and Heart. Lyon, France: World Health Organization; 2004.

2. Malpica A, Sant'Ambrogio S, Deavers MT, et al. Well-differentiated papillary mesothelioma of the female peritoneum: a clinicopathologic study of 26 cases. Am J Surg Pathol. 2012;36: $117-127$.

3. Daya D, McCaughey WT. Well-differentiated papillary mesothelioma of the peritoneum. A clinicopathologic study of 22 cases. Cancer. 1990;65:292-296.

4. Hoekstra AV, Riben MW, Frumovitz M, et al. Well-differentiated papillary mesothelioma of the peritoneum: a pathological analysis and review of the literature. Gynecol Oncol. 2005;98:161-167.

5. Kerrigan SA, Turnnir RT, Clement PB, et al. Diffuse malignant epithelial mesotheliomas of the peritoneum in women: a clinicopathologic study of 25 patients. Cancer. 2002;94:378-385.

6. Carbone M, Ferris LK, Baumann F, et al. BAP1 cancer syndrome: malignant mesothelioma, uveal and cutaneous melanoma, and MBAITs. J Transl Med. 2012;10:179.

7. Ribeiro C, Campelos S, Moura CS, et al. Well-differentiated papillary mesothelioma: clustering in a Portuguese family with a germline BAP1 mutation. Ann Oncol. 2013;24:2147-2150.

8. Cheung M, Talarchek J, Schindeler K, et al. Further evidence for germline BAP1 mutations predisposing to melanoma and malignant mesothelioma. Cancer Genet. 2013;206:206-210.

9. Chiosea S, Krasinskas A, Cagle PT, et al. Diagnostic importance of 9p21 homozygous deletion in malignant mesotheliomas. Mod Pathol. 2008;21:742-747.

10. Kong F, Zhu J, Wu J, et al. dbCRID: a database of chromosomal rearrangements in human diseases. Nucleic Acids Res. 2011;39: D895-D900.
11. Butnor KJ, Sporn TA, Hammar SP, et al. Well-differentiated papillary mesothelioma. Am J Surg Pathol. 2001;25:1304-1309.

12. Brimo F, Illei PB, Epstein JI. Mesothelioma of the tunica vaginalis: a series of eight cases with uncertain malignant potential. Mod Pathol. 2010;23:1165-1172.

13. Galateau-Sallé F, Vignaud JM, Burke L, et al. Mesopath group. Well-differentiated papillary mesothelioma of the pleura: a series of 24 cases. Am J Surg Pathol. 2004;28:534-540.

14. Goldblum J, Hart WR. Localized and diffuse mesotheliomas of the genital tract and peritoneum in women. A clinicopathologic study of nineteen true mesothelial neoplasms, other than adenomatoid tumors, multicystic mesotheliomas, and localized fibrous tumors. Am J Surg Pathol. 1995;19:1124-1137.

15. Torii I, Hashimoto M, Terada T, et al. Well-differentiated papillary mesothelioma with invasion to the chest wall. Lung Cancer. 2010;67: 244-247.

16. Trpkov K, Barr R, Kulaga A, et al. Mesothelioma of tunica vaginalis of "uncertain malignant potential" - an evolving concept: case report and review of the literature. Diagn Pathol. 2011;6:78.

17. Chen X, Sheng W, Wang J. Well-differentiated papillary mesothelioma: a clinicopathological and immunohistochemical study of 18 cases with additional observation. Histopathology. 2013;62:805-813.

18. Bürrig KF, Pfitzer P, Hort W. Well-differentiated papillary mesothelioma of the peritoneum: a borderline mesothelioma. Report of two cases and review of literature. Virchows Arch A Pathol Anat Histopathol. 1990;417:443-447.

19. Churg A, Cagle PT, Roggli VL. Tumors of the Serosal Membranes, Fascicles Series $I V$. Washington, DC: American Registry of Pathology; 2006.

20. Washimi K, Yokose T, Amitani Y, et al. Well-differentiated papillary mesothelioma, possibly giving rise to diffuse malignant mesothelioma: a case report. Pathol Int. 2013;63:220-225. 